\title{
Comparison of the Loss of Renal Function after Cold Ischemia Open Partial Nephrectomy, Warm Ischemia Laparoscopic Partial Nephrectomy and Laparoscopic Partial Nephrectomy Using Microwave Coagulation
}

\author{
Takashi Kawahara ${ }^{a}$ \\ Ryoko Sakata ${ }^{a}$ \\ Kimiko Kawaharab \\ Hiroki Ito ${ }^{a}$ \\ Yasuhide Miyoshic \\ Futoshi Sano ${ }^{a}$ \\ Noboru Nakaigawa ${ }^{a}$ \\ Hiroji Uemura ${ }^{a}$ \\ Masahiro Yao ${ }^{a}$ Yoshinobu Kubota ${ }^{a}$ \\ Kazuhide Makiyama ${ }^{a}$ \\ aDepartment of Urology, Yokohama City University Graduate School of Medicine; bepartment of Nephrology, \\ International Goodwill Hospital; cepartment of Urology, Yokohama City University Medical Center, Yokohama, Japan
}

\section{Key Words}

eGFR • Partial nephrectomy • Renal function •

Laparoscopic partial nephrectomy

\begin{abstract}
Purpose: Nephron sparing surgery is an effective surgical option in patients with renal cell carcinoma. Laparoscopic partial nephrectomy involves clamping and unclamping techniques of the renal vasculature. This study compared the postoperative renal function of partial nephrectomy using an estimation of the glomerular filtration rate (eGFR) for a Japanese population in 3 procedures; open partial nephrectomy in cold ischemia (OPN), laparoscopic partial nephrectomy in warm ischemia (LPN), and microwave coagulation using laparoscopic partial nephrectomy without ischemia (MLPN). Materials and Methods: A total of 57 patients underwent partial nephrectomy in Yokohama City University Hospital from July 2002 to July 2008. 18 of these patients underwent OPN, 17 patients received MLPN, and 22 patients had LPN. The renal function evaluation included eGFR, as recommended by The Japanese Society of Nephrology.
\end{abstract}

\section{KARGER}

Fax +4161306 1234 E-Mailkarger@karger.ch www.karger.com
Results: There was no significant difference between the 3 groups in the reduction of eGFR. eGFR loss in the OPN group was significantly higher in patients that experienced over 20 minutes of ischemia time. eGFR loss in LPN group was significantly higher in patients that experienced over 30 minutes of ischemia time. Conclusion: This study showed that all 3 procedures for small renal tumor resection were safe and effective for preserving postoperative renal function.

Copyright @ 2012 S. Karger AG, Basel

\section{Introduction}

Nephron sparing surgery (NSS) is an effective surgical option in patients with renal cell carcinoma with imperative indications, such as those with underlying renal disease, or tumors in a solitary or functionally solitary kidney [1]. In addition, NSS for patients with tumors less than $4 \mathrm{~cm}$ who have normal renal function can preserve

Takashi Kawahara, MD and Kazuhide Makiyama, MD, PhD

Department of Urology, Yokohama City University Graduate School of Medicine

3-9, Fukuura, Kanazawa

Yokohama (Japan)

E-Mail takashi_tk2001@yahoo.co.jp (Takashi Kawahara);

makiya@yokohama-cu.ac.jp (Kazuhide Makiyama) 
renal function with cancer control equivalent to radical nephrectomy $[2,3]$.

Laparoscopic surgery has been applied to partial nephrectomy for small renal tumors through retroperitoneal or transperitoneal approaches [4-6]. Laparoscopic partial nephrectomy involves clamping and unclamping techniques of the renal vasculature [7]. Laparoscopic partial nephrectomy can be performed using microwave coagulation for non-clamping surgery or clamping surgery without microwave coagulation [8].

This study compared the postoperative renal function following partial nephrectomy using an estimation of the glomerular filtration rate (eGFR) for the Japanese population using 3 procedures; open partial nephrectomy (OPN), laparoscopic partial nephrectomy (LPN), and microwave coagulation using laparoscopic partial nephrectomy (MLPN) [9].

\section{Patients and Methods}

\section{Patient Selection}

A total of 57 patients underwent partial nephrectomy in Yokohama City University Hospital from July 2002 to July 2008. Eighteen of these patients underwent OPN, 17 patients underwent MLPN, and 22 patients underwent LPN. Informed consent was obtained from all patients.

Preoperative diagnosis and clinical staging were determined by computed tomography with/without magnetic resonance imaging. The choice of surgical approach was patient and surgeon driven after the patients were informed of the benefit and risk of these 3 approaches. Patients interested in a specific approach or surgeon was treated according to their choice.

\section{$M L P N$}

Retroperitoneoscopic partial nephrectomy using a microwave tissue coagulator without renal vascular clumping was performed by the procedure reported by Murota et al. [10] and our previous report [11]. Patients were placed under general and epidural anesthesia in the lateral decubitus position with a slight flexure of the Jack knife position. A $2 \mathrm{~cm}$ skin incision was made below the 12th rib on the midaxillary line. The retroperitoneal space was extended using PD balloon systems (Tyco healthcare, Osaka, Japan). The retroperitoneal space was inflated with carbon dioxide at $10 \mathrm{mmHg}$ pressure. A $12 \mathrm{~mm}$ initial trocar was inserted, followed by a 2 nd and 3rd 5 or $12 \mathrm{~mm}$ trocar, which surrounded the initial trocar. A 4th $5 \mathrm{~mm}$ trocar was inserted based on the tumor position. A wide opening in Gerota's fascia was made along the psoas major. The kidney was separated from the surrounding peritoneal fat along the renal surface. The location and the size of the tumor were evaluated by an ultrasonographic laparoscopic probe. The renal parenchyma was incised using monopolar. Electrocoagulation was used to plot the incision line in the renal parenchyma around 8 to 10 spots. The microwave needle was inserted at the incision line along the surface of the tumor and the renal parenchyma was coagulated for $30 \mathrm{~s}$ with $65 \mathrm{~W}$ using a microwave tissue coagulator (Microtaze, Azwell, Osaka, Japan) with a needle 1.5 to $2 \mathrm{~cm}$ in length. The needle was extracted after $15 \mathrm{~s}$ of dissociation. A partial nephrectomy was performed using scissors. The tumor was put into a bag and extracted from the initial trocar site. We regularly performed electrocoagulation and compression in rare cases of bleeding that occurred in the bottom region of tumor resections. Coagulation was performed using compression, electrocoagulation and fibrin glue. A drain was established and the wounds were closed. No mobilization around the proximal renal artery and vein was performed.

\section{LPN with Warm Ischemia}

LPN was performed according to a previously reported procedure using a new parenchymal suturing technique [8]. The choice of approach depended on the location of the tumor. Anterior or upper pole apical tumors are approached transperitoneally, while posterior tumors are approached retroperitoneally. The renal artery was mobilized. The kidney was mobilized with Gerota's fascia. Gerota's fascia was opened and the renal capsule is visualized around the tumor. The renal capsule was cut by a monopolar around the tumor. Only the renal artery was clamped intracorporeally with a bulldog clamp. The tumor which was on the proximal side was clamped to both the renal artery and vein using a bulldog clamp. Partial nephrectomy was performed. Dilute indigo carmine was injected using a ureteral catheter in a retrograde fashion, followed by continuous suturing of the opened collecting system and the transected major vessel with 2-0 Vicryl on an SH needle with intracorporeal knot-tying.

A 1 Vicryl on a CT needle was used to perform the parenchymal continuous suturing. The thread was $20-30 \mathrm{~cm}$ in length, and a knot was made at the end of the thread. A polymer L size Hem-o-lok clip (Weck Closure System, Research Triangle Park, NC, USA) was attached on the proximal side of the knot. The thread was neither tightened nor crunched, and the parenchyma was sutured in a running fashion with 3 or 4 stitches without any bolster so that the renal bed remained in its natural position during the suturing. In the rare occasion the renal parenchemya was not glued, we stitched with bolster. This provided a clear view during the suturing and helped maintain accurate parenchymal sutures. Fibrin glue was dripped onto the renal bed, and the thread was tightened from far to near with suitable tension. The tightened thread was fixed with an L size Hem-o-lok, one stitch at a time. The thread can be tightened again and fixed with the Hem-o-lok if hemostasis is not confirmed after the bulldog clamp on the renal artery is removed.

\section{OPN with Cold Ischemia}

OPN was performed under general and epidural anesthesia, following occlusion of the renal artery. Cold ischemia was used in all cases after clamping of the renal artery and renal surface hypothermia was achieved using sterile ice.

\section{Measurement of Renal Function}

The levels of serum creatinine were measured before surgery, 1 month, 3 months and 6 months after surgery. All patients were followed up for more than 6 months. The renal function evaluation included serum creatinine measurement and eGFR, as recommended by The Japanese Society of Nephrology. eGFR was calculated by the formula: eGFR $=194 \mathrm{x}$ [Serum Creatinine $(\mathrm{mg} /$ dl) $]^{-1.094} \times$ [age $\left.(\mathrm{yr})^{-0.287}\right] \times(0.739$ : in female patient $)[9]$. 
Table 1. Baseline characteristic of each group

\begin{tabular}{|c|c|c|c|c|}
\hline & OPN & LPN & MLPN & $\mathrm{p}$ \\
\hline Patients, $\mathrm{n}$ & 18 & 22 & 17 & \\
\hline Age (year) & $60.0 \pm 7.0$ & $60.5 \pm 6.1$ & $54.0 \pm 5.2$ & N.S. \\
\hline Male $(\%)$ & $15(83.3 \%)$ & $15(68.2 \%)$ & $15(88.2 \%)$ & N.S. \\
\hline Right $(\%)$ & $9(50.0 \%)$ & $10(45.5 \%)$ & $11(50.0 \%)$ & N.S. \\
\hline Serum creatinin (mg/dL) & $0.85 \pm 0.16$ & $0.73 \pm 0.13$ & $0.82 \pm 0.18$ & N.S. \\
\hline $\mathrm{eGFR}\left(\mathrm{ml} / \mathrm{min} / 1.73 \mathrm{~m}^{2}\right)$ & $75.1 \pm 17.5$ & $80.9 \pm 13.6$ & $80.3 \pm 18.3$ & N.S. \\
\hline Tumor size $(\mathrm{cm})$ & $2.42 \pm 1.22$ & $1.96 \pm 0.21$ & $2.29 \pm 0.29$ & N.S. \\
\hline Estimated blood loss (mL) & $420.0 \pm 210.9$ & $100.0 \pm 104.0$ & $50.0 \pm 172.2$ & $<0.005$ \\
\hline Ischemia time (minute) & $17.0 \pm 4.4$ & $31.5 \pm 4.2$ & - & $<0.001$ \\
\hline Operative time (minute) & $185.0 \pm 18.4$ & $174.0 \pm 14.0$ & $195.0 \pm 15.3$ & N.S. \\
\hline Malignancy of pathology (\%) & $15(83.3 \%)$ & $18(81.8 \%)$ & $15(88.2 \%)$ & N.S. \\
\hline
\end{tabular}

N.S. = Not significant.

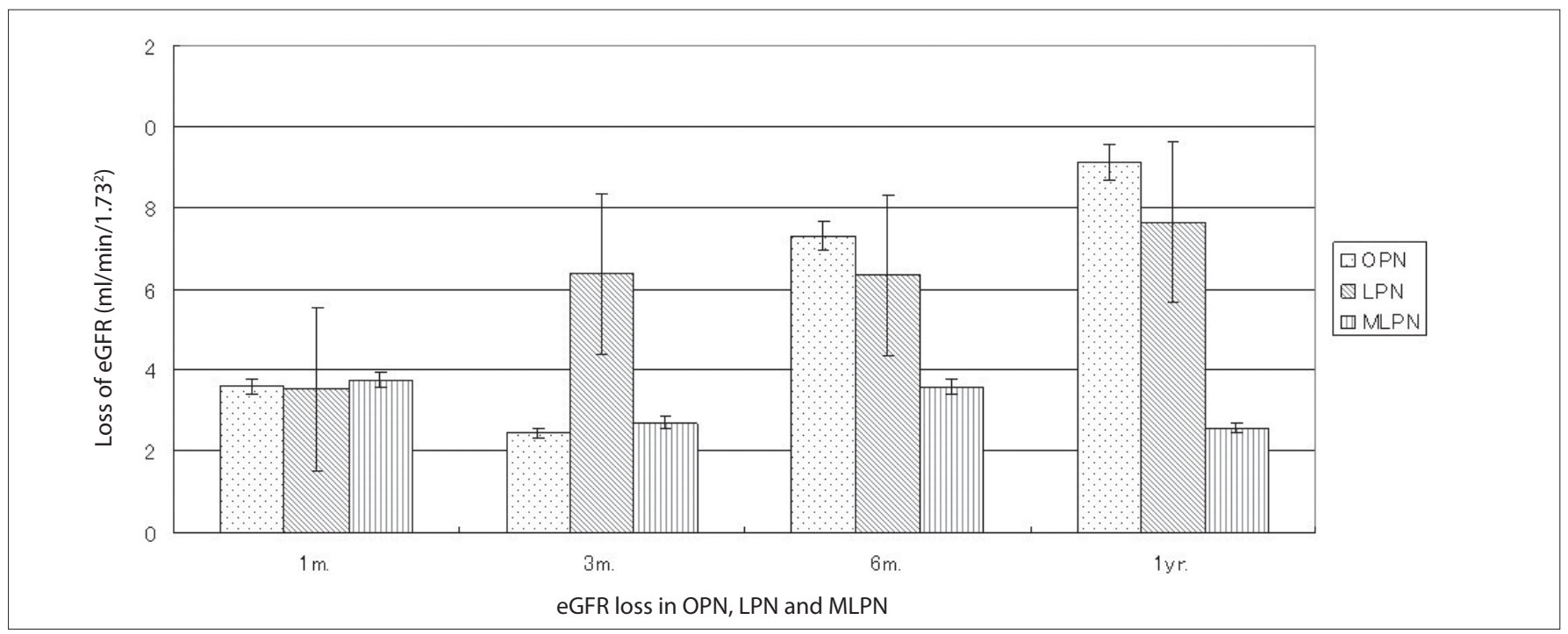

Fig. 1. eGFR loss in each group.

Statistical Analysis

All continuous variables are expressed as the mean \pm SD. The numerical data were compared by Student's t-test. A p value of 0.05 or less was considered to be significant.

\section{Results}

There was no difference in the patients' characteristics including age, gender, and side and tumor size. The his- topathological data showed no differences between each group as shown in table 1 . The duration of the procedure was $185.0 \pm 18.4$ minutes in the OPN group, $174.0 \pm$ 14.0 minutes in the LPN group and $195.0 \pm 15.3$ minutes in the MLPN group. The volume of bleeding was 420.0 $\pm 210.9 \mathrm{ml}$ in the OPN group, $100.0 \pm 104.0 \mathrm{ml}$ in the LPN group and $50.0 \pm 172.2 \mathrm{ml}$ in the MLPN group ( $\mathrm{p}$ $<0.005)$. There was no significant difference in any of the factors analyzed without ischemia time and estimated blood loss. 


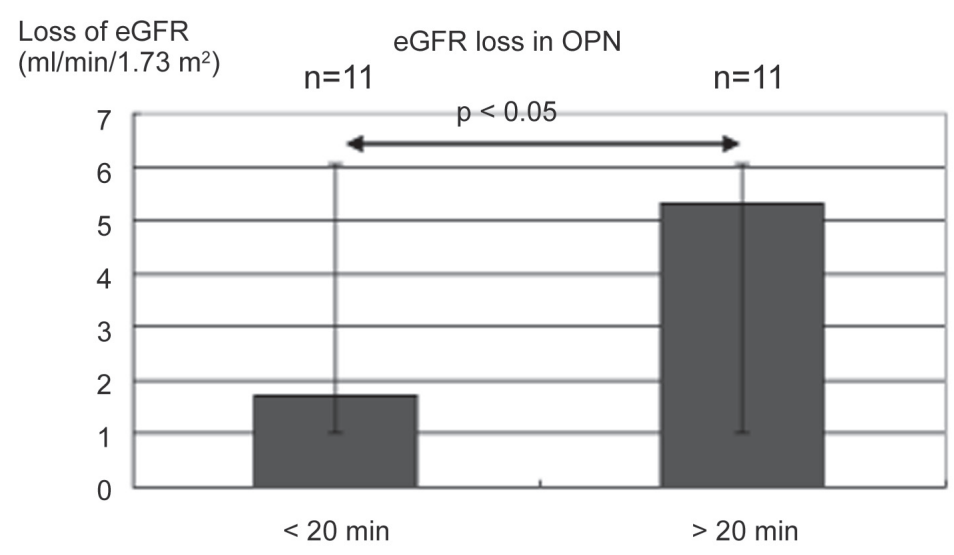

Fig. 2. Less than 20 minutes of ischemia time showed significantly lower eGFR loss in OPN.

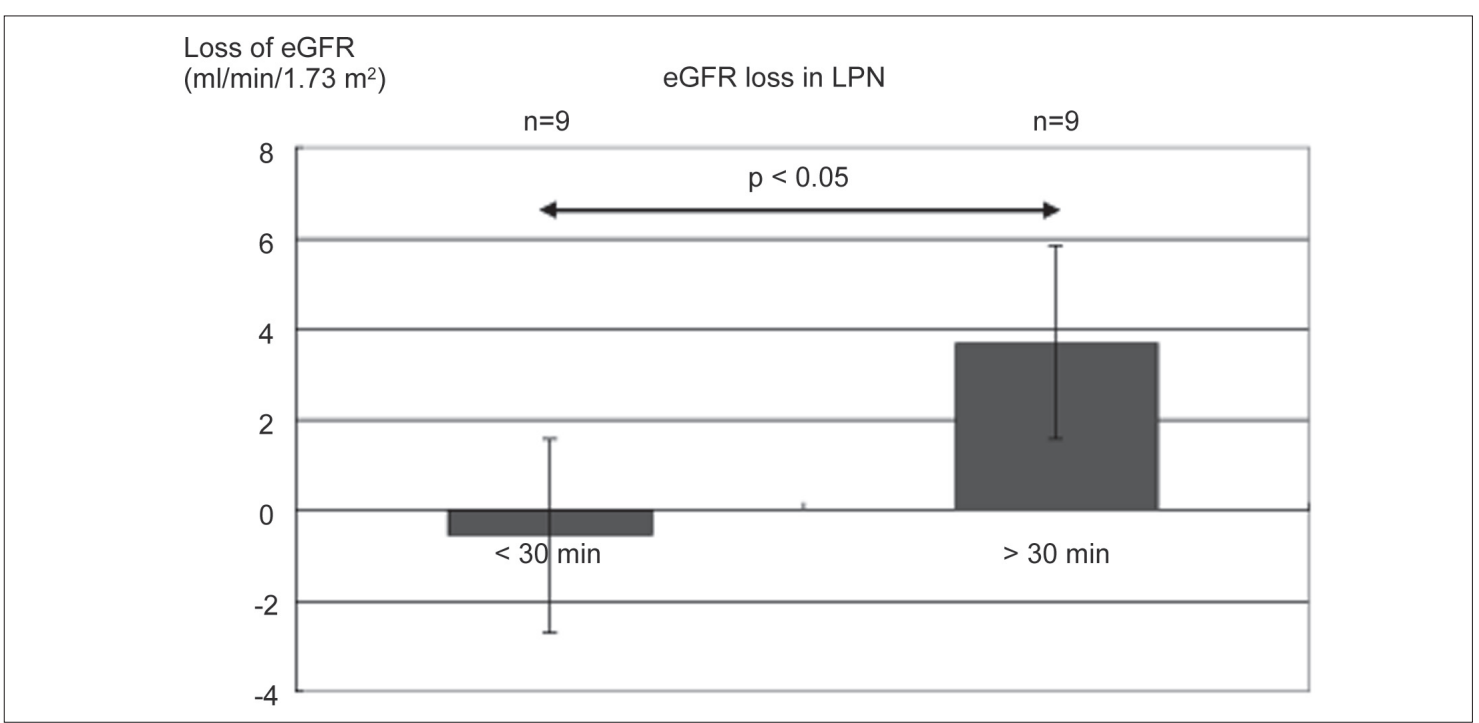

Fig. 3. Less than 30 minutes of ischemia showed significantly lower eGFR loss in LPN.

All patients were followed up for more than 6 months. The ischemia time in OPN was significantly shorter than in LPN. However, the reduction of eGFR in the three groups was not significantly different at $1,3,6$, and 12 months after surgery (fig.1). The OPN group had significantly greater eGFR loss if the patients experienced more than 20 minutes of ischemia time $(n=9)$ in comparison to less than 20 minutes of ischemia time after one month from operation $(n=9)(p<0.05)($ fig. 2$)$. The LPN group showed significantly higher eGFR loss if the patients experienced more than 30 minutes of ischemia time $(n=11)$ in comparison to less than 30 minutes of ischemia time after 3 months from operation $(n=1)(p<0.05)$ (fig. 3$)$. However, there were no significant differences in eGFR 
loss between any of the groups at any time after surgery. No positive pathological surgical margin was detected in this study. There were no major complications in any of the patients.

\section{Discussion}

The detection rate of asymptomatic small renal tumor is increasing. Partial nephrectomy is widely accepted for elective cases in which the contralateral side of the kidney is normal $[12,13]$. A previous study reported partial nephrectomy using microwave coagulation and confirmed the effectiveness for maintaining renal function and for cancer control [14].

Microwave coagulation is useful for protecting renal function because there is no ischemia [11]. However, the microwave coagulation poses a risk for inducing broad necrosis or urinary fistula in patients with relatively deep tumors. Therefore, the adaptation of partial nephrectomy using laparoscopy with clamping of the renal artery is often necessary. LPN requires a high level of surgical expertise in order to minimize ischemia time. A previous study reported the suturing technique for LPN and confirmed the effectiveness and safety of decreasing ischemia time [15]. In this study, LPN with less than $30 \mathrm{~min}$. ischemia time showed a low eGFR loss. We speculate this observed lower eGFR loss was due to the following 3 reasons: first, we incised just outside of the tumor. The next reason was the patients' conscious of drinking of large amounts of water, which we recommended to the patients at admission. The final reason was that we did not tight the renal parenchyma during the operation.

This study evaluated renal function using eGFR for a Japanese population. It would be better to assess the renal function without a solitary kidney using ${ }^{99 \mathrm{~m} T c}$ DMSA scintigraphy. On the other hand, ${ }^{99 \mathrm{~m} T c-D M S A}$ scintigraphy is expensive, so investigating the renal function at number of times periods would be is difficult. An estimation of renal function can be obtained by using the Cockcroft-Gault formula or MDRD formula [16, 17]. However, those formulas were developed for the American people, and a formula that estimates renal function for Japanese people is needed. The Japanese Society of Nephrology reported the formula for estimating GFR in Japanese people in 2008 [9]. This report is the first study that evaluated the loss of renal function for Japanese NSS patients by using the eGFR.

The loss of eGFR in MLPN one year after surgery in the current series was than that seen in other groups but not significantly. OPN and LPN groups showed higher mean eGFR loss than the other. However, the mean eGFR loss in OPN group was less than $10 \mathrm{ml} / \mathrm{min} / 1.73 \mathrm{~m}^{2}$, so no clinical influence was there whether to need dialysis. In our institute, we continuously checked the laboratory data every one year. The loss of renal function one and three months after surgery was correlated with the ischemia time in the OPN and LPN groups. Shekarriz et al. [18] reported that ischemia time less than 30 minutes shows no effect on renal function. The loss of eGFR 1 month after surgery showed significantly differences with a cutoff point of 30 minutes ischemia in LPN and 20 minutes in OPN. These data are consistent with previous reports [18]. A suturing technique that shorts the ischemia time was used in LPN, as described in 2008 [8]. This technique was used for all patients that underwent LPN in this series and results in a mean ischemia time of 31.5 minutes. These findings suggest that ischemia time less than 30 minutes might be a target time for protecting renal function in LPN for Japanese patients.

There are several limitations associated with this study. One limitation of this study was that it did not assess the split renal function. ${ }^{99 \mathrm{~m}} \mathrm{Tc}-\mathrm{DMSA}$ scintigraphy could assess the split renal function. However, evaluating the chronological changes in the renal function by $99 \mathrm{mTc}-\mathrm{DMSA}$ would be economically unfeasible. Another limitation was the small number of patients investigated and the fact that it was a retrospective study. We therefore have to establish a cutoff-point near the median clamping time (20 minutes for OPN and 30 minutes for LPN) in order to reach an equal sample size, which did not reflect the tolerance of the renal function, because kidney can tolerate longer ischemia time under cold ischemia than warm ischemia. As a result, further study is needed.

\section{Conclusion}

This study demonstrated every procedure for small renal tumor was safe and effective for preserving postoperative renal function. Postoperative renal function might be preserved if the ischemia time is limited to 30 minutes in LPN and 20 minutes in OPN. 


\section{References}

1 Fergany AF, Saad IR, Woo L, Novick AC: Open partial nephrectomy for tumor in a solitary kidney: experience with 400 cases. J Urol 2006;175:1630-1633.

$\checkmark 2$ Hafez KS, Fergany AF, Novick AC: Nephron sparing surgery for localized renal cell carcinoma: impact of tumor size on patient survival, tumor recurrence and TNM staging. J Urol 1999;162:1930-1933.

$>3$ Lee CT, Katz J, Shi W, Thaler HT, Reuter VE, Russo P: Surgical management of renal tumors $4 \mathrm{~cm}$ or less in a contemporary cohort. J Urol 2000;163:730-736.

4 Ito H, Makiyama K, Kawahara T, Sano F, Murakami T, Hayashi N, Miyoshi Y, Nakaigawa N, Yao M, Kubota Y: Retroperitoneoscopic radical nephrectomy with a small incision for renal cell carcinoma: comparison with the conventional method. J Negat Results Biomed 2011;10:11.

$\checkmark 5$ Winfield HN, Donovan JF, Godet AS, Clayman RV: Laparoscopic partial nephrectomy: initial case report for benign disease. J Endourol 1993; 7:521-526.

-6 Gill IS, Desai MM, Kaouk JH, Meraney AM, Murphy DP, Sung GT, Novick AC: Laparoscopic partial nephrectomy for renal tumor: duplicating open surgical techniques. J Urol 2002;167:469-477.

7 Guillonneau B, Bermudez H, Gholami S, El Fettouh H, Gupta R, Adorno Rosa J, Baumert H, Cathelineau X, Fromont G, Vallancien G: Laparoscopic partial nephrectomy for renal tumor: single center experience comparing clamping and no clamping techniques of the renal vasculature. J Urol 2003;169:483-486.
8 Makiyama K, Nakaigawa N, Miyoshi Y, Murakami $\mathrm{T}$, Yao $\mathrm{M}$, Kubota Y: Improvement on parenchymal suturing technique in laparoscopic partial nephrectomy. Int J Urol 2008; 15:854-855.

-9 Matsuo S, Imai E, Horio M, Yasuda Y, Tomita K, Nitta K, Yamagata K, Tomino Y, Yokoyama H, Hishida A: Revised equations for estimated GFR from serum creatinine in Japan. Am J Kidney Dis 2009;53:982-992.

10 Murota T, Kawakita M, Oguchi N, Shimada O, Danno S, Fujita I, Matsuda T: Retroperitoneoscopic partial nephrectomy using microwave coagulation for small renal tumors. Eur Urol 2002;41:540-545.

11 Makiyama K, Nakaigawa N, Miyoshi y, Murakami T, Yao M, Kubota Y: Retroperitoneoscopic partial nephrectomy using microwave tissue coagulator without renal vascular clumping for small renal tumors. Jpn J Endourol ESWL 2007;20:84-88.

12 Sharma N, O'Hara J, Novick AC, Lieber M, Remer EM, Herts BR: Correlation between loss of renal function and loss of renal volume after partial nephrectomy for tumor in a solitary kidney. J Urol 2008;179:1284-1288.

13 Colombo JR Jr, Haber GP, Gill IS: Laparoscopic partial nephrectomy in patients with compromised renal function. Urology 2008 ; 71:1043-1048.
14 Porpiglia F, Renard J, Billia M, Musso F, Volpe A, Burruni R, Terrone C, Colla L, Piccoli G, Podio V, Scarpa RM: Is renal warm ischemia over 30 minutes during laparoscopic partial nephrectomy possible? One-year results of a prospective study. Eur Urol 2007; 52:1170-1178.

15 Desai MM, Gill IS, Ramani AP, Spaliviero M, Rybicki L, Kaouk JH: The impact of warm ischaemia on renal function after laparoscopic partial nephrectomy. BJU Int 2005; 95:377-383.

16 Cockcroft DW, Gault MH: Prediction of creatinine clearance from serum creatinine. Nephron 1976;16:31-41.

17 Levey AS, Bosch JP, Lewis JB, Greene T, Rogers N, Roth D: A more accurate method to estimate glomerular filtration rate from serum creatinine: a new prediction equation. Modification of Diet in Renal Disease Study Group. Ann Intern Med 1999;130:461-470.

18 Shekarriz B, Shah G, Upadhyay J: Impact of temporary hilar clamping during laparoscopic partial nephrectomy on postoperative renal function: a prospective study. J Urol 2004; 172:54-57. 